\title{
A Piezoelectric Cryogenic Heat Switch
}

\author{
Amir E. Jahromi, ${ }^{1, a}$ and Dan F. Sullivan ${ }^{1, b}$ \\ ${ }^{1}$ NASA Goddard Space Flight Center, Cryogenics and Fluids branch code 552, Greenbelt, MD, U.S.A.
}

\begin{abstract}
We have measured the thermal conductance of a mechanical heat switch actuated by a piezoelectric positioner, the PZHS (PieZo electric Heat Switch), at cryogenic temperatures. The thermal conductance of the PZHS was measured between $4 \mathrm{~K}$ and $10 \mathrm{~K}$, and on/off conductance ratios greater than 100 were achieved when the positioner applied its maximum force of $8 \mathrm{~N}$. We discuss the advantages of using this system in cryogenic applications, and estimate the ultimate performance of an optimized PZHS.
\end{abstract}

Keywords: Heat switch, Thermal switch, mechanical heat switch, Piezoelectric heat switch, Adiabatic Demagnetization Refrigerator, liquid helium thermal switch

\section{INTRODUCTION}

Heat switches are used to control the transfer of heat between objects at different temperatures, and are particularly important in cryogenic environments. Applications in this context include minimizing the parasitic heat load from a cryocooler in a redundant refrigeration system [1], connecting instruments to and from a cryo-radiator [2], and transferring heat from a salt pill in an adiabatic demagnetization refrigerator (ADR) [3]. Many types of cryogenic heat switches exist, including gas gap, mechanically actuated, superconducting, and fluid-loop based [4]. In this work, we describe the construction and characterize the performance of a novel mechanical cryogenic heat switch actuated by a piezoelectric positioner, the PZHS.

Our motivation in developing the PZHS stems from the fact that many NASA science missions require cryogenic capabilities; consequently, heat switches often play a critical role in the success of these experiments. Since efficient photon detection tends to be greatly enhanced at lower temperatures $(\mathrm{T} \leq 1 \mathrm{~K})$ [5], ADRs are firmly entrenched as NASA's preferred technology for detectors operating in this temperature regime. Current state of the art ADRs use multiple gas gap heat switches to transfer heat from the salt pill during the magnetization (heat rejection) stage of the refrigeration cycle. Although these heat switches have demonstrated the performance required, for example, by the Astro-H mission

a) Contact email: amir.e.jahromi@nasa.gov

b) Contact email: dan.f.sullivan@nasa.gov 
[6], their use is limited to either very low $(\mathrm{T}<0.3 \mathrm{~K})$ or relatively high $(\mathrm{T} \sim 5 \mathrm{~K})$ cryogenic temperatures. In addition, the hermetic joints required to contain the ${ }^{3} \mathrm{He}$ exchange gas within the switch housing have shown vulnerability to the mechanical stresses associated with thermal cycling and vibration. The PZHS is thus an attractive alternative technology to a gas gap heat switch, since this device has an essentially unlimited range of cryogenic operating temperatures, is mechanically robust, and is free from hermetic sealing requirements. In the remainder of this paper we will discuss the design and construction of the PZHS test apparatus, the measurements carried out to characterize the system, our experimental results, and plans for future work.

\section{PZHS Design and Construction}

The principle of operation of the PZHS is elegantly simple; our experimental apparatus for demonstrating this device is shown in Fig. 1. Two high-conductivity metallic plates act as independent thermal reservoirs, with one mounted to a support structure and the other fastened to the mobile stage of a piezoelectric positioner (an attocube ANPz101) [7]. When the positioner is energized with a series of positive voltage ramps, the lower plate moves upwards until mechanical contact is established with the upper plate. In this configuration, the switch is closed, and heat transfer occurs between the two plates. After the desired heat transfer is complete, energizing the positioner with negative voltage ramps moves it downwards until mechanical contact is lost between the plates, leaving the switch open.

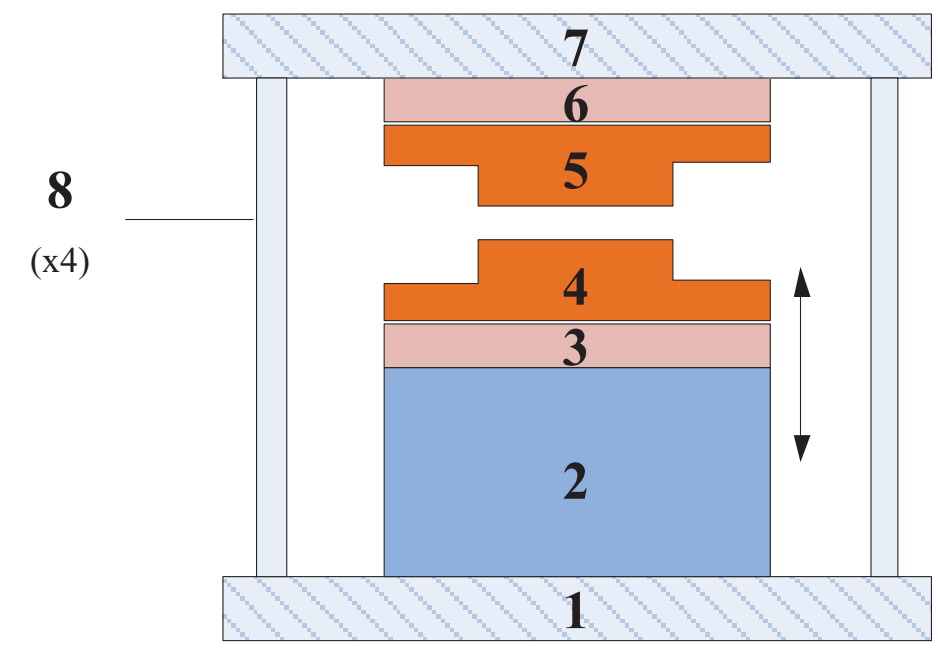

FIG. 1. A schematic of the PZHS design; 1 Base support plate 2 Piezoelectric positioner 3 Lower insulator 4 Lower conductor 5 Upper conductor 6 Upper insulator 7 Top support plate 8 Structure columns (x4) 
The key figure of merit for this device is the switching ratio, $S$, defined by:

$$
S \equiv \frac{k_{c}}{k_{o}}
$$

where $k_{c}$ and $k_{o}$ are the thermal conductance of the PZHS when closed and opened, respectively. Proper engineering of a heat switch thus consists of maximizing $k_{c}$ while minimizing $k_{o}$. When the switch is open, during steady state operation, parasitic heat flows from the high to low temperature reservoir via the structure columns. To mitigate this, and consequently minimize $k_{o}$, the columns were constructed from G-10 hollow rods, and the plates were mounted on Vespel SP1 insulators. To maximize $k_{c}$, the upper and lower plates were made from ultra high purity (99.999\%) copper, with a contact area of 1.45 $\times 1.45 \mathrm{~cm}^{2}$.

With the expectation that the PZHS performance would be limited by the joint conductance, we paid particular attention to the preparation of the plate surfaces, striving to make them as flat and as parallel as possible. This would have the effect of increasing the effective PZHS contact area and thus enhancing $k_{c}$. Our method is shown in Fig. 2. The switch plates were bolted to a larger, hollowed out block, with walls slightly higher than the plate itself. This assembly was then placed on a lapping table, and the switch surfaces were sanded by pouring isopropanol alcohol on sand paper of progressively finer paper grits, culminating in the use of a special cotton impregnated with metal polish. Once the surfaces were immaculate, the switch plates were cleaned and gold plated with $\sim 1 \mu \mathrm{m}$ of Au. The plating prevents tarnishing of the copper, and also acts as a "cushion" to the switch surfaces, further enhancing the effective contact area. Table 1 summarizes the properties of the main components of the PZHS.

Table 1 Properties and dimensions of major components of our PZHS

\begin{tabular}{|c|c|c|}
\hline Parts & Material & Dimension \\
\hline Upper and Lower conductor & $99.999 \%$ pure copper & $\begin{array}{l}\text { Contact area square of } \\
1.45 \mathrm{~cm} \text { on each side }\end{array}$ \\
\hline Upper and Lower insulator & Vespel SP-1 & $7 \mathrm{~mm}$ thick square block \\
\hline Support columns & G-10 & $\begin{array}{c}4.3 \mathrm{~cm} \text { long, } 6.3 \mathrm{~mm} \text { OD, } \\
3.2 \mathrm{~mm} \mathrm{ID}\end{array}$ \\
\hline
\end{tabular}




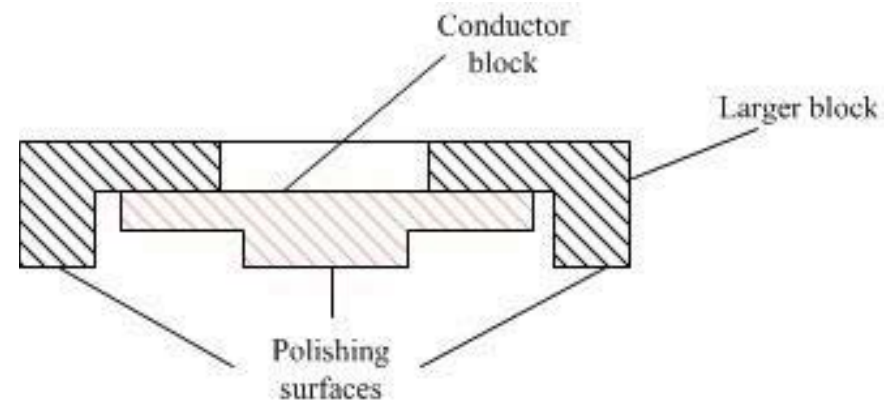

FIG 2. Lapping method for the copper plates to ensure flat, parallel and smooth PZHS mating surfaces

In order to establish the desired temperature of the reservoirs independently, a small thermometer and heater were mounted on each plate, and the temperatures were maintained using a PID controller. To allow for reasonable thermal time constants ( minutes), thin electrically insulated copper wires were attached from each reservoir to the cold stage of the test cryostat, a cryocooler with a base temperature of $\sim 3 \mathrm{~K}$. Contact between the plates was checked using a two-wire resistance measurement. With the switch open, no continuity was observed; with the switch closed, continuity was confirmed, with a measured resistance of $\sim 80 \Omega$. We note that all of our closed switch data was obtained with the positioner applying its maximum specified force of $8 \mathrm{~N}$. A photograph of the complete PZHS assembly is shown in Fig. 3.

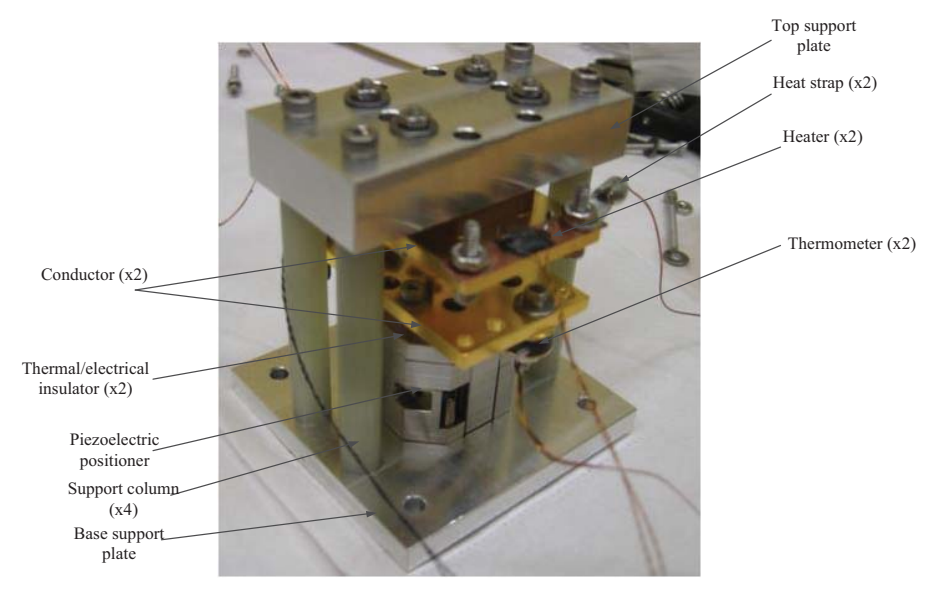


Figure 3. PZHS test assembly. The assembly was tested in a cryostat with base temperature $\sim 3 \mathrm{~K}$.

\section{Experiments and results}

Figure 4 shows a simplified schematic of the conductance network in our PZHS test apparatus. The upper switch plate, lower switch plate, and cryocooler cold plate temperatures are labeled $T_{U}, T_{L}$, and $T_{b}$, respectively. The conductance of the mechanical joint is $k_{c}$, while the upper switch plate is connected to the cold plate through a conductance $k_{U}$, and the lower switch plate is connected to the cold plate through a conductance $k_{L}$. With the switch open, $k_{c}=0$, and $k_{U}$ and $k_{L}$ can be determined as a function of temperature by setting $T_{U}$ and $T_{L}$ equal:

$$
\text { if } T_{U}=T_{L}\left\{\begin{array}{l}
\dot{q}_{U}=k_{U}\left(T_{U}-T_{b}\right) \\
\dot{q}_{L}=k_{L}\left(T_{L}-T_{b}\right)
\end{array}\right.
$$

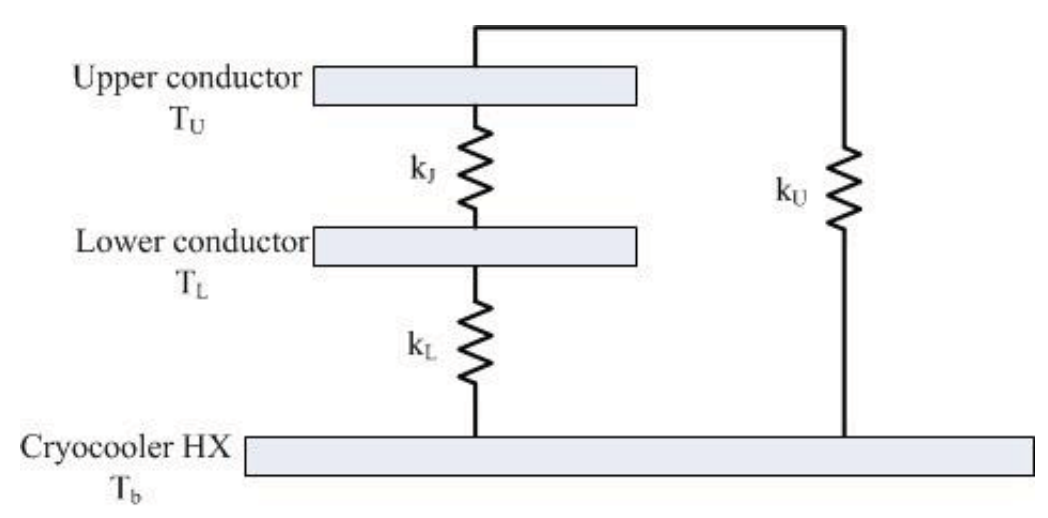

Figure 4. A simplified conductance network of the PZHS test apparatus.

We observed that both $k_{U}$ and $k_{L}$ were linearly dependent on reservoir temperature, with best-fit results (in $\mathrm{mW} / \mathrm{K}$ ) of $k_{U}=3.2 \times 10^{-6}+\left(6.4 \times 10^{-6}\right) T_{U}$ and $k_{L}=5.4 \times 10^{-6}+\left(4.3 \times 10^{-6}\right) T_{L}$. After measuring the conductance from the reservoirs to the cold plate, the joint conductance $k_{c}$ was determined by closing the switch, independently controlling the temperature of each plate, and applying the following equations: 


$$
\begin{aligned}
& \text { if } T_{U}=T_{L}+\delta T\left\{\begin{array}{l}
\dot{q}_{U}=k_{U}\left(T_{U}-T_{b}\right)+k_{J}\left(T_{U}-T_{L}\right) \\
\dot{q}_{L}=k_{L}\left(T_{L}-T_{b}\right)
\end{array}\right. \\
& \text { if } T_{U}+\delta T=T_{L}\left\{\begin{array}{l}
\dot{q}_{U}=k_{U}\left(T_{U}-T_{b}\right) \\
\dot{q}_{L}=k_{L}\left(T_{L}-T_{b}\right)+k_{J}\left(T_{L}-T_{U}\right)
\end{array}\right.
\end{aligned}
$$

Solving the set of equations in Eq. (3) yields the conductance of the mechanical joint, $k_{c}$. We plot the results of our experiments in Fig. 5 for temperatures from $4 \mathrm{~K}$ to $10 \mathrm{~K}$. A conservative estimate of the PZHS switching ratio at $4 \mathrm{~K}$ can be obtained by setting $k_{o}=k_{U} \sim 0.028 \mathrm{~mW} / \mathrm{K}$, which yields $\mathrm{S} \sim 100$. We note that the thermal conductance for a solid piece of copper at $4 \mathrm{~K}$ with the same dimensions as our switch plates is $62 \mathrm{~W} / \mathrm{K}$, which represents the theoretical upper limit of performance for the PZHS. A more realistic estimate, based on studies of bolted mechanical joints (Cryogenics 44(5): 293-299 (2004)), suggests a limit of $\sim 10 \mathrm{~W} / \mathrm{K}$. Thus, as anticipated, the PZHS conductance is limited by $k_{c}$, and future work should focus on methods to improve this parameter.

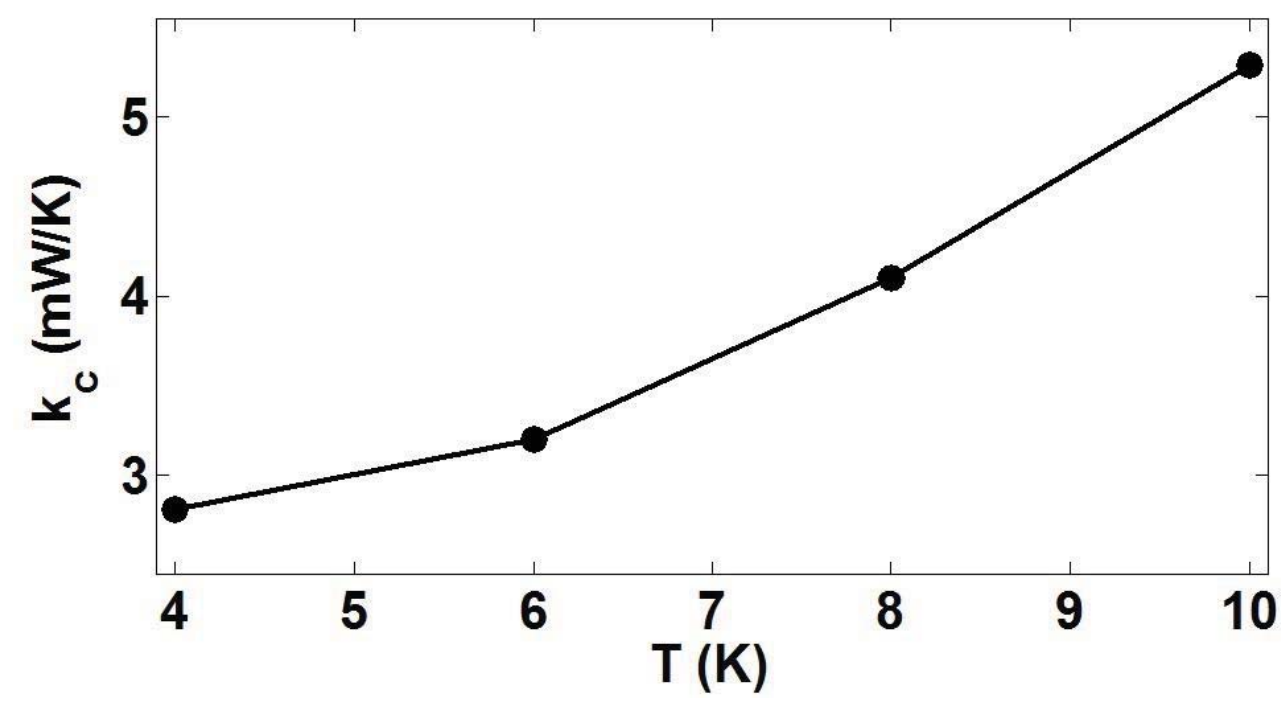

FIG 5. Thermal conductance of the PZHS vs. temperature.

\section{Conclusions}

We have demonstrated a mechanical heat switch, the PZHS, actuated by piezoelectric elements for use in cryogenic applications, with a switching ratio $\mathrm{S} \sim 100$ at $4 \mathrm{~K}$. In principle, such a heat switch would be sufficiently versatile to operate at all cryogenic temperatures of interest to NASA. The PZHS 
has the potential for significant improvement by using piezoelectric positioners with larger clamping forces, in addition to spring loading on a thermal reservoir. We hope to incorporate these and other improvements in a subsequent version of the PZHS.

\section{REFERENCES}

${ }^{1}$ Van Oost, S., Bekaert, G., Bhatti Sabca, R.S., Scull, S., and Jewell, C., “A Heat Switch for Space Cryocooler Applications," Proceedings of the 4th European Symposium on Space Environmental and Control Systems, Florence, Italy, 21-24 October, 1991 (ESA SP-324.December 1991).

${ }^{2}$ Bugby, D.C., Cepeda-Rizo, J., and Rodriguez, J. I., “Thermal switching Cryogenic Heat Pipe,” Proceedings of the 16th International Cryocoolers Conference, Boulder, CO, 2011.

${ }^{3}$ Shirron, Peter J., et al. "Design of a 3-stage ADR for the soft x-ray spectrometer instrument on the ASTRO-H mission." SPIE Astronomical Telescopes and Instrumentation: Observational Frontiers of Astronomy for the New Decade. International Society for Optics and Photonics, 2010.

${ }^{4}$ M. Donabedian, Spacecraft Thermal Control Handbook Vol. II: Cryogenics. El Segundo, CA; 2003.

${ }^{5}$ Glass, I. S. (1999). Handbook of Infrared Astronomy. New York: Cambridge University Press. ISBN 0-521-63311-7.

${ }^{6}$ M. O. Kimball. P. J. Shirron, AIP Conf. Proc. 1434, 853 (2012); doi 10.1063/1.4707000.

${ }^{7}$ Attocube Systems AG., Königinstrasse 11a. 80539, München. Germany. 